\title{
Complete percutaneous repair after valvular surgery
}

\author{
Jose Carlos Moreno-Samos, Ignacio Cruz-Gonzalez, Manuel Barreiro-Perez, \\ Rocio Gonzalez-Ferreiro, Monica Fuertes-Barahona, Pedro Luis Sanchez-Fernandez \\ University Hospital of Salamanca, Institute of Biomedical Research in Salamanca (IBSAL), \\ Faculty of Medicine, University of Salamanca, CIBERCV, Salamanca, Spain
}

A 54-year-old woman with previous valvular surgery and permanent atrial fibrillation was admitted due to congestive heart failure.

In 2005, she underwent open-heart surgery when mitral valve was replaced (31-mm On-X mitral valve [CryoLife, Inc.]). Also a tricuspid annuloplasty (34-mm Edwards MC3 ring [Edwards Lifesciences]) and a left atrial appendage (LAA) ligation were completed.

A transesophageal echocardiography showed a lateral paravalvular leak $(12 \times 6 \mathrm{~mm})$ in the prosthetic mitral valve (Fig. 1A). Percutaneous closure of the paravalvular leak was performed (Fig. 1B, C) with an Amplatzer Vascular Plug III device $(12 \times 5 \mathrm{~mm})$ (St. Jude Medical) with no residual regurgitation detected (Fig. 1D, E).

Subsequent to this, the patient was admitted because of recurrent cardioembolic strokes despite optimal treatment with warfarin.
Cardiac imaging evaluation detected a residual communication between the left atrium and previously ligated LAA (Fig. 1F) and a percutaneous closure was decided upon.

After a LAA angiogram identified incomplete ligation (Fig. 1G), a $16 \mathrm{~mm}$ Amplatzer Vascular Plug II device (St. Jude Medical) was placed via 8 Fr Mullins sheath which sealed the orifice with no residual flow (Fig. 1H, I).

Incomplete LAA closure following surgical ligation occurs in $30-40 \%$ of cases and is associated with a significantly increased risk of thrombus formation/thromboembolism. Although, percutaneous closure of incomplete ligation has been previously described, according to available research, this is the first reported case of a complete percutaneous repair of two complications after valvular surgery.

Conflict of interest: Dr. Ignacio Cruz-Gonzalez is proctor for St. Jude Medical. The other authors have no conflicts of interest to disclose. 


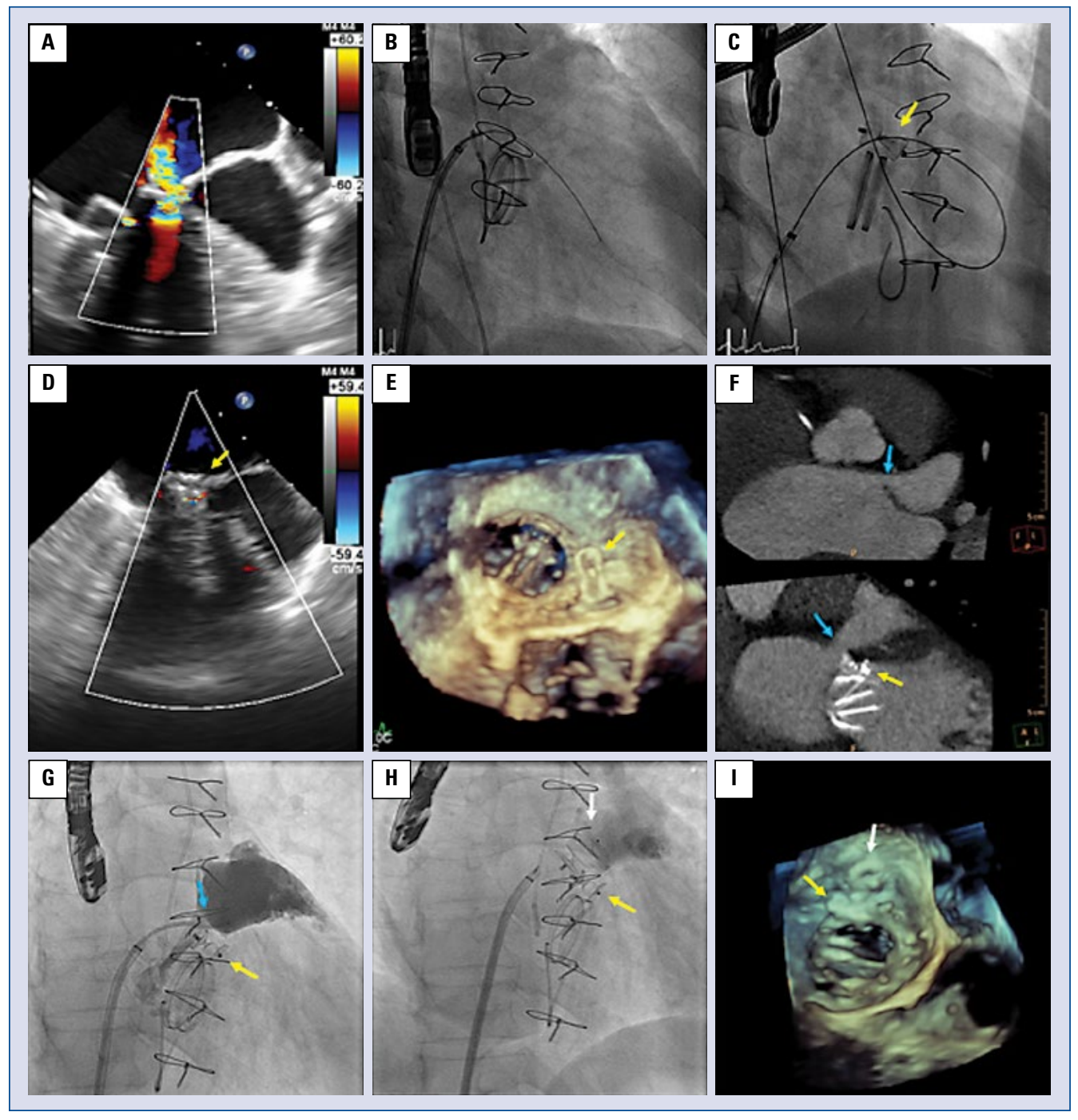

Figure 1. A. Two-dimensional transesophageal echocardiography (2D TEE) view of paravalvular regurgitation; B. Fluoro image: the leak was crossed with a straight wire; C. Fluoro image: after venoarterial loop was created, an Amplatzer Vascular Plug III device (AVPIII) $12 \times 5-\mathrm{mm}$ device (yellow arrow) was deployed; D. 2D TEE image showing no paravalvular regurgitation; E. 3D TEE image showing AVPIII device; F. Computed tomography image showing incomplete left atrial appendage (LAA) ligation (blue arrow); G. Fluoro image: baseline left atrial appendage angiogram; H. Fluoro image: after deployment of an AVPII 16 mm device (white arrow); I. 3D TEE image showing the position of both devices. 